\title{
A Concise, Catalyst-Free Synthesis of Davis' Oxaziridines using Sodium Hypochlorite
}

\author{
Saori Kitagawa \\ Hiromitsu Mori \\ Tatsuya Odagiri ${ }^{b}$ \\ Katsuya Suzukia \\ You Kikkawa ${ }^{a}$ \\ Rie Osugi \\ Shinobu Takizawa ${ }^{\text {(1) }}$ \\ Yoshikazu Kimurad ${ }^{\text {(B) }}$ \\ Masayuki Kirihara*a (10) \\ a Department of Materials and Life Science, Shizuoka Institute of Science and Technology, \\ 2200-2 Toyosawa, Fukuroi, Shizuoka 437-8555, Japan \\ kirihara.masayuki@sist.ac.jp \\ ${ }^{b}$ R\&D Department of Chemicals, Nippon Light Metal Company, Ltd., Kambara, Shimizu- \\ ku, Shizuoka 421-3203, Japan \\ c The Institute of Scientific and Industrial Research (ISIR), Osaka University, Mihogaoka, \\ Ibaraki-shi, Osaka 567-0047, Japan \\ ${ }^{d}$ Research and Development Department, Iharanikkei Chemical Industry Co. Ltd., Kamba- \\ ra, Shimizu-ku, Shizuoka 421-3203, Japan
}

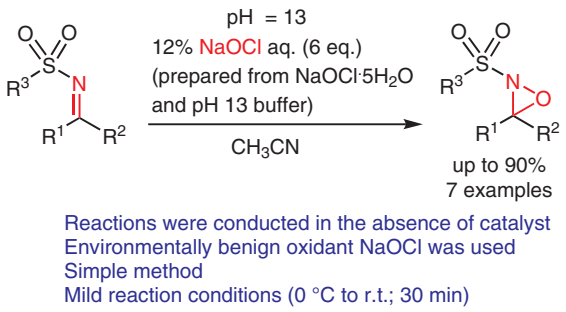

( $m$-chloroperbenzoic acid, ${ }^{2 \mathrm{a}, 3 \mathrm{a}-\mathrm{d}}$ Oxone ${ }^{\mathrm{TM}},{ }^{2 \mathrm{~b}}$ hydrogen peroxide or cumene hydroperoxide (CHP) ${ }^{3 \mathrm{~h}}$ with a catalyst ${ }^{3 \mathrm{e}-\mathrm{g}}$ ) have been used for this purpose (Scheme 1).

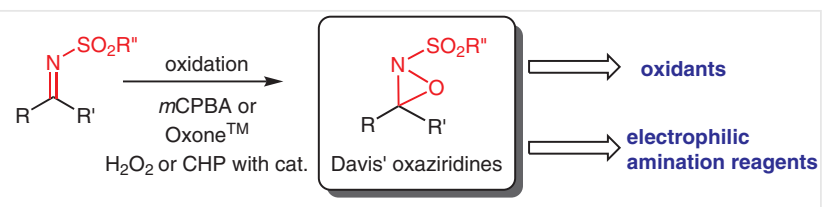

Scheme 1

Sodium hypochlorite $(\mathrm{NaOCl})$ is an ideal oxidant in organic synthesis, ${ }^{4}$ because it produces only non-toxic sodium chloride $(\mathrm{NaCl})$ as a by-product following oxidation, and commercial aqueous $\mathrm{NaOCl}$ is non-explosive and inexpensive. Recently, we found that stable, crystalline sodium hypochlorite pentahydrate $\left(\mathrm{NaOCl} \cdot 5 \mathrm{H}_{2} \mathrm{O}\right),{ }^{5}$ which is now commercially available from several companies, is a very useful oxidant for the nitroxy radical-catalyzed oxidation of alcohols, ${ }^{5 b, 5 c}$ as well as the oxidation of organosulfur compounds. ${ }^{5 \mathrm{~d}, 5 \mathrm{e}}$ However neither of these practical, environmentally friendly oxidants, aq. $\mathrm{NaOCl}$ or $\mathrm{NaOCl} \cdot 5 \mathrm{H}_{2} \mathrm{O}$, have been used for the preparation of Davis' oxaziridines. In this paper, we report the concise, catalyst-free preparation of Davis' oxaziridines via reaction of $\mathrm{N}$-sulfonyl imines in aqueous acetonitrile with $\mathrm{NaOCl}$ under basic conditions (Scheme 2). 

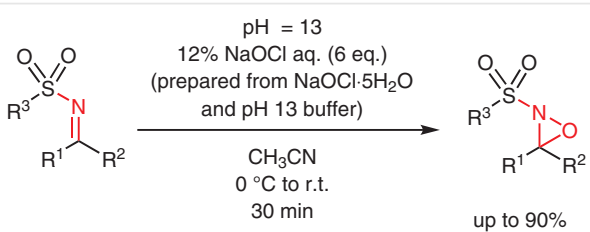

Scheme 2

In initial optimization studies, $N$-tosylimine (1a) derived from benzaldehyde (3a) was chosen as a substrate, and treated with conventional $12 \%$ aqueous $\mathrm{NaOCl}$ in several solvents (Table 1). The desired oxaziridine $\mathbf{2 a}$ was produced efficiently in acetonitrile (entry 1 ). On the other hand, hydrolysis of the imine 1a predominated in other solvents, regenerating $\mathbf{3 a}$ (entries 2-4).

Table 1 Solvent Effect for the reaction of $1 \mathbf{a}$ with $\mathrm{NaOCl}$

\begin{tabular}{|c|c|c|c|c|c|}
\hline & 1a $(1 \mathrm{mmol})$ & $\begin{array}{l}12 \% \mathrm{NaOCl} \text { aq. }{ }^{a} \\
\text { (12 eq.) } \\
\text { solvent }(5 \mathrm{~mL}) \\
0{ }^{\circ} \mathrm{C} \text { to r.t. } \\
0.5 \mathrm{~h}\end{array}$ & & & \\
\hline \multirow[t]{3}{*}{ Entry } & Solvent & Time (h) & \multicolumn{3}{|c|}{${ }^{1} \mathrm{H}$ NMR ratio } \\
\hline & & & $1 \mathrm{a}$ & $2 a$ & $3 a^{b}$ \\
\hline & & & \multicolumn{3}{|c|}{ (SM) (oxaziridine) } \\
\hline 1 & $\mathrm{CH}_{3} \mathrm{CN}$ & 0.5 & 0 & 85 & 15 \\
\hline 2 & $t-\mathrm{BuOH}$ & 5 & 1 & 5 & 94 \\
\hline 3 & $\mathrm{CH}_{2} \mathrm{Cl}_{2}$ & 24 & 8 & 57 & 35 \\
\hline 4 & toluene & 24 & 0 & 3 & 97 \\
\hline
\end{tabular}

a Conventional aqueous solution was used.

b $p$-Toluenesulfonylamide was produced as the side-product from hydration of 1 a.

In our effort to explore the use of environmentally benign oxidants, $\mathrm{NaOCl} \cdot 5 \mathrm{H}_{2} \mathrm{O}$ proved to be superior to conventional, commercial aqueous $\mathrm{NaOCl}$ in this type of reaction. Therefore, the reaction of $\mathbf{1 a}$ with $12 \%$ aqueous $\mathrm{NaOCl}$, freshly prepared from crystalline $\mathrm{NaOCl} \cdot 5 \mathrm{H}_{2} \mathrm{O}$, was examined (Table 2). Contrary to our expectation, the desired oxaziridine 2a was obtained in low yield, accompanied by a large amount of benzaldehyde 3a, produced from the hydration of $1 \mathbf{a}$ (entry 2) as with the alternative solvent above.
Table 2 Comparison of Conventional vs. Freshly Prepared 12\% aq. NaOCl

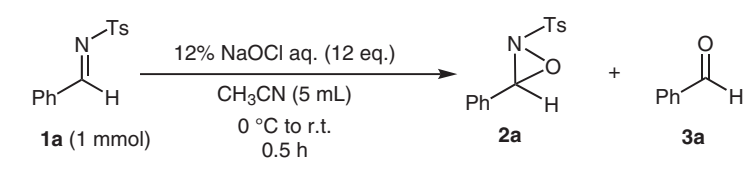

\begin{tabular}{llccc}
\hline Entry & $12 \% \mathrm{NaOCl}$ aq. & $\mathrm{pH}$ & \multicolumn{2}{c}{${ }^{1} \mathrm{H}$ NMR ratio } \\
& & & 2a & 3a \\
\hline 1 & conventional aq. solution & 13 & 85 & 15 \\
2 & prepared from $\mathrm{NaOCl} \cdot 5 \mathrm{H}_{2} \mathrm{O}$ & 11 & 10 & 90 \\
\hline
\end{tabular}

The main difference between conventional aqueous $\mathrm{NaOCl}$ and an aqueous solution prepared from $\mathrm{NaOCl} \cdot 5 \mathrm{H}_{2} \mathrm{O}$ crystals is the $\mathrm{pH}^{5}$ As shown in Table 2, the $\mathrm{pH}$ of the former was 13 , and the $\mathrm{pH}$ of the latter was 11 . In our previous work $^{5 b, 5 c}$ on the nitroxy radical-catalyzed oxidation of alcohols and the catalyst-free oxidation of sulfides to sulfoxides with $\mathrm{NaOCl} \cdot 5 \mathrm{H}_{2} \mathrm{O}$, we observed that the $\mathrm{pH}$ of the aqueous $\mathrm{NaOCl}$ solutions dramatically influenced oxidation reactivity. Therefore, the reactivity of the $12 \% \mathrm{NaOCl}$ aqueous solutions prepared from $\mathrm{NaOCl} \cdot 5 \mathrm{H}_{2} \mathrm{O}$ was evaluated while altering the $\mathrm{pH}$ with $\mathrm{HCl}$ or $\mathrm{NaOH}$ (Table 3).

Table 3 Reactivity of aq $12 \% \mathrm{NaOCl}$ with Varying $\mathrm{pH}$

\begin{tabular}{|c|c|c|c|c|c|}
\hline & & mol) & $\begin{array}{l}\quad \mathrm{pH}=\mathrm{X} \\
12 \% \mathrm{NaOCl} \text { aq. (12 eq.) } \\
\text { (Prepared from } \mathrm{NaOCl} \cdot 5 \mathrm{H}_{2} \mathrm{O} \\
\text { or conventional solution ) }\end{array}$ & $2 a+3$ & \\
\hline & & (1701) & $\begin{array}{c}\mathrm{CH}_{3} \mathrm{CN}(5 \mathrm{~mL}) \\
0{ }^{\circ} \mathrm{C} \text { to r.t. } \\
30 \mathrm{~min}\end{array}$ & & \\
\hline Entry & $\mathrm{pH}$ & $12 \%$ & $\mathrm{NaOCl}$ aq. & ${ }^{1} \mathrm{H} \mathrm{N}$ & ratio \\
\hline & & & & $2 a$ & $3 a$ \\
\hline 1 & $14^{\mathrm{a}}$ & & pared from $\mathrm{NaOCl} \cdot 5 \mathrm{H}_{2} \mathrm{O}$ & 68 & 32 \\
\hline 2 & $14^{\mathrm{a}}$ & & nventional solution & 81 & 19 \\
\hline 3 & $13^{\mathrm{a}}$ & & pared from $\mathrm{NaOCl} \cdot 5 \mathrm{H}_{2} \mathrm{O}$ & 87 & 13 \\
\hline 4 & $13^{\mathrm{a}}$ & & nventional solution & 85 & 15 \\
\hline 5 & $12^{\mathrm{a}}$ & & pared from $\mathrm{NaOCl} \cdot 5 \mathrm{H}_{2} \mathrm{O}$ & 26 & 74 \\
\hline 6 & $12^{\mathrm{b}}$ & & nventional solution & 30 & $70^{c}$ \\
\hline 7 & $10^{\mathrm{b}}$ & & pared from $\mathrm{NaOCl} \cdot 5 \mathrm{H}_{2} \mathrm{O}$ & 0 & $100^{c}$ \\
\hline 8 & $10^{\mathrm{b}}$ & Co & nventional solution & 0 & $0^{c}$ \\
\hline
\end{tabular}

As we had proposed, the reactivity of $\mathbf{1 a}$ toward $\mathrm{NaOCl}$ depended on the $\mathrm{pH}$ of the reaction mixture. A pH of 13 optimized the outcome (entries 3 and 4), and 2a was obtained in good isolated yields (71\% and 69\%) as shown in Scheme 3. 


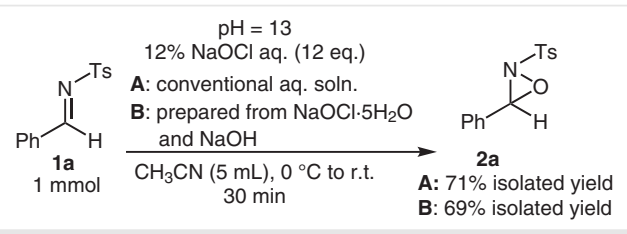

Scheme 3

Further screening of the reaction conditions revealed that 6 equivalents of $12 \%$ aqueous $\mathrm{NaOCl}$ solution, prepared from $\mathrm{NaOCl} \cdot 5 \mathrm{H}_{2} \mathrm{O}$ with commercial $\mathrm{pH} 13$ buffer $(\mathrm{KCl}-$ $\mathrm{NaOH}$ ) in acetonitrile, afforded 1a in $90 \%$ isolated yield (Scheme 4).

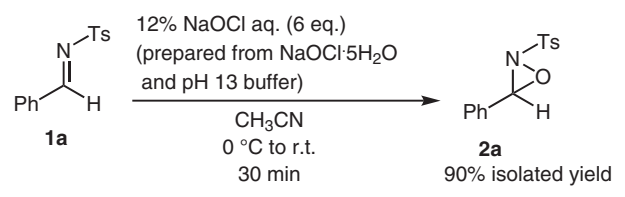

Scheme 4

Several sulfonylimines 1 were then treated with aqueous $\mathrm{NaOCl}$ under the optimized conditions using $\mathrm{CH}_{3} \mathrm{CN}$ as the solvent, including the optically active substrate $\mathbf{1 g}$ (Table 4 ). In most cases, the corresponding oxadirizine 2 was obtained in moderate to high yield. In the case of $\mathbf{1 f}$, the desired 2 f was obtained in only $4 \%$ yield accompanied by several unidentified by-products. Since the reaction conditions were basic, the acidic methyl proton of $\mathbf{1 f}$ might be removed, and the resulting anion 1f' converted into several by-products (Scheme 5).

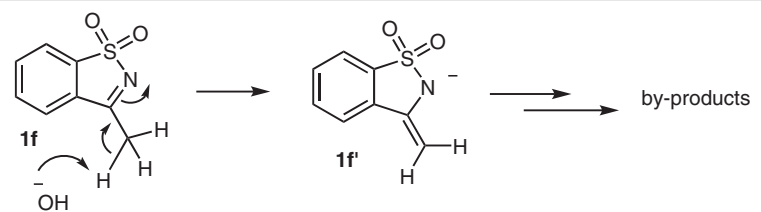

Scheme 5

A plausible reaction mechanism for the formation of $\mathbf{2}$ is depicted in Scheme 6. A hypochlorite anion attacks the imine carbon of $\mathbf{1}$ to produce intermediate $\mathbf{A}$ and then the amide anion attacks the oxygen atom to produce the oxaziridine 2. Strongly basic conditions are required to prevent the hydrolysis of $\mathbf{1}$ to the corresponding aldehyde.

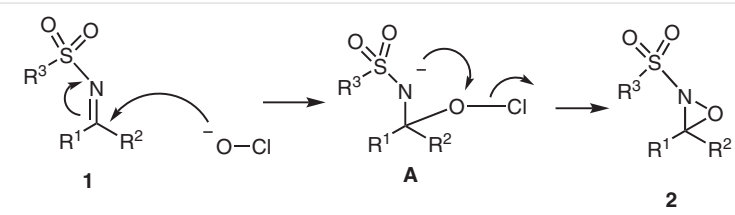

Scheme 6
Table 4 Reaction of 1 with $\mathrm{NaOCl}$ in $\mathrm{CH}_{3} \mathrm{CN}$

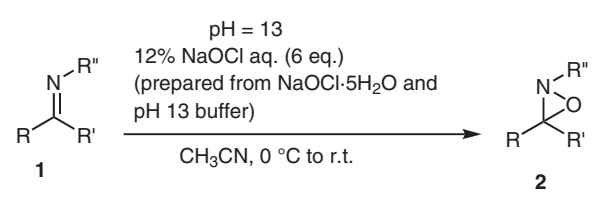

\begin{tabular}{llll}
\hline & 2 & Time (min) & Yield (\%) \\
\hline 1 &
\end{tabular}<smiles>[Al]N=Cc1ccc2ccccc2c1</smiles><smiles></smiles>

$67^{\mathrm{b}}$<smiles>O=S1(=O)N=C(c2ccccc2)c2ccccc21</smiles><smiles>O=S1(=O)c2ccccc2C2(c3ccccc3)ON21</smiles><smiles>O=S1(=O)N=C(c2ccc(Cl)cc2)c2ccccc21</smiles><smiles>O=S1(=O)c2ccccc2C2(c3ccc(Cl)cc3)ON21</smiles><smiles>O=S1(=O)N=C(c2cccc(Cl)c2)c2ccccc21</smiles><smiles>O=S1(=O)c2ccccc2C2(c3cccc(Cl)c3)ON21</smiles><smiles>CC1=NS(=O)(=O)c2ccccc21</smiles><smiles>CC12ON1S(=O)(=O)c1ccccc12</smiles>

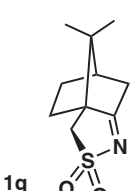<smiles>CC1(C)C2CCCC13CN(O2)S(=O)(=O)C3</smiles>

30 $66^{a}$

\footnotetext{
a Isolated yield.

${ }^{\mathrm{b}}{ }^{\mathrm{H}}$ NMR yield using an internal standard (dimethyl sulfone).
}

Oxaziridines 2, prepared from the reaction of $\mathbf{1}$ with $\mathrm{NaOCl}$, can be used as oxidants. We confirmed that the $\alpha$ hydroxylation of 2-methyl-1-tetralone using $\mathbf{2 a}$ or $\mathbf{2 g}$ as the oxidant provided results that tallied with those reported in the literature. ${ }^{2}$ 
In conclusion, synthetically useful $\mathrm{N}$-sulfonyloxaziridines (Davis' oxaziridines) $\mathbf{2}$ can be synthesized from reaction of the corresponding $\mathrm{N}$-sulfonylimine $\mathbf{1}$ with aqueous $\mathrm{NaOCl}$ in acetonitrile without need for a catalyst. Strongly basic conditions ( $\mathrm{pH} 13$ ) are required to obtain the products in high yields. ${ }^{6}$ Although both $\mathrm{NaOCl} \cdot 5 \mathrm{H}_{2} \mathrm{O}$ and conventional aq. $\mathrm{NaOCl}$ can be used as the oxidant, $\mathrm{NaOCl} \cdot 5 \mathrm{H}_{2} \mathrm{O}$ is recommended because, due to the instability of conventional aq. $\mathrm{NaOCl}$, we observed that the target compounds are not always obtained in high yields.

All reagents were purchased from Nacalai Tesque, Wako Pure Chemicals Industries, Kanto Kagaku, Kishida Reagents Chemical Co., Tokyo Chemical Industry, or Aldrich, and used without further purification. Melting points were measured with a Yanaco micro melting point apparatus (MP-J3) and are uncorrected. NMR spectra were recorded with a JEOL (JNM-EX400) spectrometer as solutions in $\mathrm{CDCl}_{3}$ using TMS or the residual $\mathrm{CHCl}_{3}$ peak as an internal standard. IR spectra were recorded with a JASCO IR-8300 FT-IR spectrophotometer. Mass spectra were recorded with a Shimadzu GCMS-QP1100EX spectrometer. Specific rotations were measured with a JASCO DIP-370 polarimeter. All of the sulfonyl imines 1 were prepared according to reported methods (1a, $\left.{ }^{2 \mathrm{a}} \mathbf{1 b},{ }^{3 \mathrm{a}} \mathbf{1 c},{ }^{\text {a }} \mathbf{1 d},{ }^{3 \mathrm{a}} \mathbf{1 e},{ }^{\text {a }} \mathbf{1 f},{ }^{3 \mathrm{a}} \mathbf{1 g}^{2 \mathrm{c}}\right)$. NMR yields were determined using dimethyl sulfone as an internal standard. All of the products $\mathbf{2}$ and $\mathbf{5}$ are known compounds, and ${ }^{1} \mathrm{H}$ and ${ }^{13} \mathrm{C}$ NMR data of the products obtained in this study are identical to those reported.

\section{Preparation of 2; Typical Procedure}

An aqueous $\mathrm{NaOCl}$ solution prepared from $\mathrm{NaOCl} \cdot 5 \mathrm{H}_{2} \mathrm{O}(987 \mathrm{mg}, 6.0$ $\mathrm{mmol})$ and $\mathrm{pH} 13$ buffer solution $(\mathrm{KCl}-\mathrm{NaOH})(50 \mathrm{~mL})$ was added to a stirred solution of $\mathbf{1 a}(260 \mathrm{mg}, 1.0 \mathrm{mmol})$ in acetonitrile $(10 \mathrm{~mL})$ at $0{ }^{\circ} \mathrm{C}$. The resulting mixture was stirred at r.t. for $30 \mathrm{~min}$. Water $(30$ $\mathrm{mL}$ ) was added to the reaction mixture, and the mixture was extracted with EtOAc $(3 \times 30 \mathrm{~mL})$. The extract was washed with brine, dried with anhydrous magnesium sulfate, filtered and evaporated. The residue was purified by silica-gel column chromatography using hexane/EtOAc (20:1) as an eluent to obtain pure $\mathbf{2 a}$ (250 mg, 90\%) as colorless crystals.

\section{3-Phenyl-2-tosyl-1,2-oxaziridine (2a) $)^{2 a}$}

Colorless crystals: $\mathrm{mp} 87-92{ }^{\circ} \mathrm{C}\left(\right.$ lit. $\left.^{2 \mathrm{a}} 87^{\circ} \mathrm{C}\right)$.

${ }^{1} \mathrm{H} \mathrm{NMR}\left(\mathrm{CDCl}_{3}\right): \delta=7.86(\mathrm{~d}, J=8.4 \mathrm{~Hz}, 2 \mathrm{H}), 7.33-7.42(\mathrm{~m}, 7 \mathrm{H}), 5.38$ (s, $1 \mathrm{H}), 2.43(\mathrm{~s}, 3 \mathrm{H})$.

${ }^{13} \mathrm{C} \mathrm{NMR}\left(\mathrm{CDCl}_{3}\right): \delta=145.39,130.37,129.51,129.03,128.39,127.70$, 127.20, 75.30, 28.68, 20.81 .

MS: $m / z=259\left(\mathrm{M}^{+}-\mathrm{O}\right), 155\left(\mathrm{M}^{+}-\mathrm{C}_{7} \mathrm{H}_{6} \mathrm{ON}\right), 91\left(\mathrm{M}^{+}-\mathrm{C}_{7} \mathrm{H}_{6} \mathrm{O}_{3} \mathrm{NS}\right)$.

IR (neat): 2924, 2856, 1596, 1351, 1170, 1087, 807, 712, 569, 543, $421 \mathrm{~cm}^{-1}$.

\section{3-(Naphthalene-2-yl)-2-tosyl-1,2-oxaziridine (2b) ${ }^{3 \mathrm{a}}$}

According to the typical procedure, $\mathbf{1 b}(312.5 \mathrm{mg}, 1.01 \mathrm{mmol})$ was treated with aq. $\mathrm{NaOCl}$ to provide a crude product $(277.6 \mathrm{mg})$ containing $\mathbf{2 b}$ (67\%, ${ }^{1} \mathrm{H}$ NMR yield using dimethyl sulfone as an internal standard). All the peaks shown below appeared in the ${ }^{1} \mathrm{H}$ and ${ }^{13} \mathrm{C}$ NMR spectra of the crude product.
${ }^{1} \mathrm{H} \mathrm{NMR}\left(\mathrm{CDCl}_{3}\right): \delta=8.03(\mathrm{brs}, 1 \mathrm{H}), 7.96(\mathrm{~d}, J=8.4 \mathrm{~Hz}, 2 \mathrm{H}), 7.89-7.84$ $(\mathrm{m}, 3 \mathrm{H}), 7.57-7.52(\mathrm{~m}, 2 \mathrm{H}), 7.45(\mathrm{~d}, J=8.0 \mathrm{~Hz}, 2 \mathrm{H}), 7.40(\mathrm{dd}, J=8.8$, $2.0 \mathrm{~Hz}, 1 \mathrm{H}), 5.61(\mathrm{~s}, 1 \mathrm{H}), 2.50(\mathrm{~s}, 3 \mathrm{H})$.

${ }^{13} \mathrm{C} \mathrm{NMR}\left(\mathrm{CDCl}_{3}\right): \delta=146.43,142.20,134.72,132.57,131.50,130.07$, $129.75,129.45,128.86,128.33,127.92,127,88,127.64,126.82$, $123.45,21.86$.

7b-Phenyl-7bH-benzo[d][1,2]oxazireno[2,3-b]isothiazol2-3,3-dioxide (2c) $)^{3 a}$

According to the representative procedure, 2c (190 mg, 73\%) was obtained from 1c $(242 \mathrm{mg}, 1.00 \mathrm{mmol})$.

Colorless crystals: $\mathrm{mp} 97-99^{\circ} \mathrm{C}\left(\right.$ lit. $\left.^{2 \mathrm{i}} 105-106^{\circ} \mathrm{C}\right)$.

${ }^{1} \mathrm{H} \mathrm{NMR}\left(\mathrm{CDCl}_{3}\right): \delta=7.88(\mathrm{dd}, J=6.8,1.2 \mathrm{~Hz}, 1 \mathrm{H}), 7.79-7.71(\mathrm{~m}, 2 \mathrm{H})$, 7.65-7.50 (m, $6 \mathrm{H})$.

${ }^{13} \mathrm{C}$ NMR $\left(\mathrm{CDCl}_{3}\right): \delta=134.57,134.44,133.84,132.79,131.34,129.00$, $128.07,128.04,127.87,124.13,85.25$.

7b-(4-Chlorophenyl)-7bH-benzo[d][1,2]oxazireno[2,3-b]isothiazol2-3,3-dioxide (2d) ${ }^{3 \mathrm{a}}$

According to the representative procedure, 2d (237 mg, 81\%) was obtained from 1d $(276 \mathrm{mg}, 1.00 \mathrm{mmol})$

White solid; mp $140-143{ }^{\circ} \mathrm{C}$ (lit. ${ }^{3 a} 144-145{ }^{\circ} \mathrm{C}$ ).

${ }^{1} \mathrm{H}$ NMR $\left(\mathrm{CDCl}_{3}\right): \delta=7.88(\mathrm{dd}, J=6.4,1.6 \mathrm{~Hz}, 1 \mathrm{H}), 7.81-7.73(\mathrm{~m}, 2 \mathrm{H})$, 7.63-7.61 (dd, $J=6.8,0.8 \mathrm{~Hz}, 1 \mathrm{H}), 7.57-7.49(\mathrm{~m}, 4 \mathrm{H})$.

${ }^{13} \mathrm{C}$ NMR $\left(\mathrm{CDCl}_{3}\right): \delta=137.75,134.41,133.98,133.95,133.00,129.51$, $129.36,127.82,126.42,124.24,84.68$.

7b-(3-Chlorophenyl)-7bH-benzo[d][1,2]oxazireno[2,3-b]isothiazol2-3,3-dioxide (2e $)^{3 \mathrm{a}}$

According to the representative procedure, $2 \mathbf{d}(237 \mathrm{mg}, 81 \%)$ was obtained from 1d $(276 \mathrm{mg}, 1.00 \mathrm{mmol})$.

Pale-orange solid; $\mathrm{mp} 124-126^{\circ} \mathrm{C}$ (lit. ${ }^{3 a} 125-126^{\circ} \mathrm{C}$ ).

${ }^{1} \mathrm{H} \mathrm{NMR}\left(\mathrm{CDCl}_{3}\right): \delta=7.90-7.87(\mathrm{~m}, 1 \mathrm{H}), 7.82-7.74(\mathrm{~m}, 2 \mathrm{H}), 7.64-7.59$ ( $\mathrm{m}, 2 \mathrm{H}), 7.56$ (td, $J=7.6,1.6 \mathrm{~Hz}, 1 \mathrm{H}), 7.52-7.45(\mathrm{~m}, 2 \mathrm{H})$.

${ }^{13} \mathrm{C}$ NMR $\left(\mathrm{CDCl}_{3}\right): \delta=135.22,134.35,134.06,133.76,133.06,131.61$, $130.40,129.93,128.15,127.86,126.29,124.24,84.38$.

7b-Methyl-7bH-benzo[d][1,2] oxazireno[2,3-b]isothiazol2-3,3-dioxide (2f) $)^{3 \mathrm{a}}$

According to the representative procedure, 1 f $(181.5 \mathrm{mg}, 1.00 \mathrm{mmol})$ was treated with aq. $\mathrm{NaOCl}$ to provide a crude product $(28.0 \mathrm{mg})$ containing $2 \mathbf{f}\left(4 \%,{ }^{1} \mathrm{H}\right.$ NMR yield using dimethyl sulfone as an internal standard). All the peaks shown below appeared in the ${ }^{1} \mathrm{H}$ and ${ }^{13} \mathrm{C}$ NMR spectra of the crude product.

${ }^{1} \mathrm{H} \mathrm{NMR}\left(\mathrm{CDCl}_{3}\right): \delta=7.80-7.71(\mathrm{~m}, 4 \mathrm{H}), 2.14(\mathrm{~s}, 3 \mathrm{H})$.

${ }^{13} \mathrm{C} \mathrm{NMR}\left(\mathrm{CDCl}_{3}\right): \delta=135.09,134.06,133.29,132.56,125.57,123.84$, 83.91, 15.57 .

(+)-(4aR,7R)-9,9-Dimethyltetrahydro-4H-4a,7-methanobenzo[c][1,2]oxazireno[2,3-b]isothiazole 3,3-Dioxide (2g) $)^{2 c}$

According to the representative procedure, $\mathbf{2 g}$ (151 $\mathrm{mg}, 66 \%)$ was obtained from $1 \mathrm{~g}$ (210 $\mathrm{mg}, 1.00 \mathrm{mmol})$.

Colorless crystals; $\mathrm{mp} 167-172{ }^{\circ} \mathrm{C}\left(\right.$ lit. $\left.^{2 \mathrm{c}} 165-167{ }^{\circ} \mathrm{C}\right) ;[\alpha]_{\mathrm{D}}+44.6\left(\mathrm{CHCl}_{3}\right.$, c 1.9) $\left\{\right.$ lit. $^{2 \mathrm{c}}[\alpha]_{\mathrm{D}}+44.6\left(\mathrm{CHCl}_{3}\right.$, c 1.8) .

${ }^{1} \mathrm{H} \mathrm{NMR}\left(\mathrm{CDCl}_{3}\right): \delta=3.10$ and $3.27\left(\mathrm{AB}\right.$ quartet, $\left.J=14 \mathrm{~Hz}, 2 \mathrm{H}, \mathrm{CH}_{2}-\mathrm{SO}_{2}\right)$, $2.64(\mathrm{~m}, 1 \mathrm{H}), 1.50-2.13(\mathrm{~m}, 6 \mathrm{H}), 1.18(\mathrm{~s}, 3 \mathrm{H}), 1.03(\mathrm{~s}, 3 \mathrm{H})$. 
${ }^{13} \mathrm{C} \mathrm{NMR}\left(\mathrm{CDCl}_{3}\right): \delta=98.77,54.06,48.32,45.80,33.64,28.39,26.56$, $20.55,19.50$.

\section{Funding Information}

This work was performed under the Cooperative Research Program of the 'Network Joint Research Center for Materials and Devices'.

\section{References and Notes}

(1) For recent reviews, see: (a) Williamson, K. S.; Michaelis, D. J.; Yoon, T. P. Chem. Rev. 2014, 114, 8016. (b) Sala, G. D.; Lattanzi, A. ACS Catal. I 2014, 4, 1234.

(2) (a) Davis, F. A.; Stringer, O. D. J. Org. Chem. 1982, 47, 1774. (b) Davis, F. A.; Chattopadhyay, S.; Towson, J. C.; Lal, S.; Reddy, T. J. Org. Chem. 1988, 53, 2087. (c) Davis, F. A.; Towson, J. C.; Weismiller, M. C.; Lal, S.; Carroll, P. J. J. Am. Chem. Soc. 1988, 110, 8477. (d) Davis, F. A.; Sheppard, A. C.; Chen, B.; Haque, M. S. J. Am. Chem. Soc. 1990, 112, 6679. (e) Davis, F. A.; Weismiller, M. C. J. Org. Chem. 1990, 55, 3715. (f) Davis, F. A.; Sheppard, A. C. Tetrahedron 1989, 45, 5703. (g) Davis, F. A.; Chen, B. C. Chem. Rev. 1992, 92, 919. (h) Petrov, V. A.; Resnati, G. Chem. Rev. 1996, 96, 1809. (i) Narasaka, K.; Ukaji, Y.; Watanabe, K. Bull Chem. Soc. Jpn. 1987, 96, 1457.

(3) Asymmetric syntheses of oxaziridines: (a) Takizawa, S.; Kishi, K.; Abozeid, M. A.; Murai, K.; Fujioka, H.; Sasaia, H. Org. Biomol. Chem. 2016, 14, 761. (b) Lykke, L.; Rodríguez-Escrich, C.; Jørgensen, K. A. J. Am. Chem. Soc. 2011, 133, 14932. (c) Zhang, T.;
He, W.; Zhao, X.; Jin, Y. Tetrahedron 2013, 69, 7416. (d) Jin, Y.; Zhang, T.; Zhang, W.; Chang, S.; Feng, B. Chirality 2014, 26, 150. (e) Uraguchi, D.; Tsutsumi, R.; Ooi, T. J. Am. Chem. Soc. 2013, 135, 8161. (f) Tsutsumi, R.; Kim, S.; Uraguchi, D.; Ooi, T. Synthesis 2014, 871. (g) Uraguchi, D.; Tsutsumi, R.; Ooi, T. Tetrahedron 2014, 70, 1691. (h) Olivares-Romero, J. L.; Li, Z.; Yamamoto, H. J. Am. Chem. Soc. 2012, 134, 5440.

(4) Galvin, J. M.; Jacobsen, E. N.; Palucki, M.; Frederick, M. O. e-EROS 2013, DOI: 10.1002/047084289X.rs084.pub3 ; and references cited therein.

(5) (a) Kirihara, M.; Okada, T.; Sugiyama, Y.; Akiyoshi, M.; Matsunaga, T.; Kimura, Y. Org. Process Res. Dev. 2017, 21, 1925. (b) Okada, T.; Asawa, T.; Sugiyama, Y.; Kirihara, M.; Iwai, T.; Kimura, Y. Synlett 2014, 25, 596. (c) Okada, T.; Asawa, T.; Sugiyama, Y.; Iwai, T.; Kirihara, M.; Kimura, Y. Tetrahedron 2016, 72, 2818. (d) Okada, T.; Matsumuro, H.; Kitagawa, S.; Iwai, T.; Yamazaki, K.; Kinoshita, Y.; Kimura, Y.; Kirihara, M. Synlett 2015, 26, 2547. (e) Okada, T.; Matsumuro, H.; Iwai, T.; Kitagawa, S.; Yamazaki, K.; Akiyama, T.; Asawa, T.; Sugiyama, Y.; Kimura, Y.; Kirihara, M. Chem. Lett. 2015, 44, 185.

(6) Given that addition of $10 \mathrm{~mol} \%$ tetrabutylammonium hydroxide $\left(\mathrm{Bu}_{4} \mathrm{NOH}\right)$ accelerated the oxidation reaction in a biphasic solvent system using trifluoromethylbenzene (benzotrifluoride, BTF), the reaction might proceed enantioselectively if an optically pure alkylammonium salt was employed. As a preliminary result, the desired product 2a was obtained with 7\% ee when the reaction was conducted using $\mathrm{N}$-benzylcinchonidinium chloride. 